\title{
Medical Management of Gestational Diabetes
}

\author{
Ayesha Anwar1 ${ }^{*}$, Kashif Ahmad1, Evdokia Karagianni'1, Stephen Lindow ${ }^{2}$ \\ ${ }^{1}$ North Cumbria University Hospitals, NHS Trust, Carlisle, UK \\ ${ }^{2}$ Sidra Medical and Research Center, Weill Cornell Medical College in Qatar, Doha, Qatar \\ Email: *ayeshakashif1@hotmail.com
}

How to cite this paper: Anwar, A., Ahmad, K., Karagianni, E. and Lindow, S. (2018) Medical Management of Gestational Diabetes. Open Journal of Obstetrics and Gynecology, 8, 400-407.

https://doi.org/10.4236/ojog.2018.84045

Received: March 7, 2018

Accepted: April 22, 2018

Published: April 25, 2018

Copyright $\odot 2018$ by authors and Scientific Research Publishing Inc. This work is licensed under the Creative Commons Attribution International License (CC BY 4.0).

http://creativecommons.org/licenses/by/4.0/

\begin{abstract}
Gestational diabetes (GDM) is defined as carbohydrate intolerance that begins or is first recognized during pregnancy. The prevalence of GDM is on the rise due to increasing trends of obesity in females of child bearing age as well as due to increasing maternal age. Women at higher risk should be routinely screened of the condition and offered appropriate management in order to avoid fetal and maternal complications. GDM is associated with higher incidence of maternal and fetal complications during pregnancy and labour. There is a significant relationship between GDM and prediabetic states as well as high risk of developing type 2 diabetes mellitus. The aim of management is to keep glycaemic levels within specific targets to avoid adverse outcomes. Improved health related quality of life has been linked with treatment of gestational diabetes with active lifestyle, glucose monitoring and use of insulin. Historically, non pharmacological intervention and insulin have been the main approaches in management of GDM, though recently there is an emerging evidence of use of oral hypoglycemic agents. In this article we have reviewed the existing evidence and recommendations in management of GDM.
\end{abstract}

\section{Keywords}

Gestational Diabetes, Pregnancy, Management

\section{Introduction}

Gestational diabetes (GDM) is defined as carbohydrate intolerance that begins or is first recognized during pregnancy (WHO 2017). Gestational Diabetes is estimated to be present in $5 \%-15 \%$ of pregnant women, though these figures vary greatly among different ethnic groups [1]. It is growing health concern worldwide. As there is a strong association of GDM and maternal obesity, the prevalence of GDM is on rise with epidemic of growing obesity and widespread sedentary 
lifestyle [2] [3]. Advanced maternal age is considered as an additional factor of the recent increasing prevalence of GDM.

GDM is associated with higher incidence of maternal and fetal complications during pregnancy and labour. It is characterized by increased risk of miscarriage, hypertensive disorders including preeclampsia and HELLP syndrome, preterm birth and stillbirth. Fetal growth disorders are common, mainly in terms of macrosomia, which is in its turn associated with an increased risk of operative delivery, caesarean section, shoulder dystocia and birth trauma. Children born from mothers with GDM also experience increased rates of perinatal hypoglycemia, jaundice, respiratory distress and need for admission to intensive care unit. It is interesting that in babies born from mothers with GDM there is a long-term risk of developing diabetes and obesity in childhood and adult life [4]. Furthermore, it is reported that pregnant women with GDM have an at least seven-fold increased risk of developing diabetes later in life [5].

However, adequate control of maternal blood glucose levels has been shown to reduce the rates of adverse perinatal outcomes and potentially long term consequences [6]. Research has led to recommendations that attempts should be made to keep glucose levels in pregnant women to near normal [1].

\section{Risk Assessment, Screening and Diagnosis of Gestational Diabetes}

Effective risk assessment screening and early diagnosis are important to minimize the adverse outcome linked to GDM. NICE recommends prescreening counseling to patients, explaining clearly that diagnosis of gestational diabetes will lead to increased monitoring, and may lead to increased interventions, during both pregnancy and labour.

The high risk patient offered for screening are women with, BMI above $30 \mathrm{~kg} / \mathrm{m}^{2}$, previous gestational diabetes, previous macrosomic baby (weighing above the $90^{\text {th }}$ centile), family history of diabetes (first-degree relative with diabetes) and minority ethnic family origin with a high prevalence of diabetes [7]. Women with a previous history of gestational diabetes are offered early selfmonitoring of blood glucose or a 2-hour $75 \mathrm{~g}$ oral glucose tolerance test (OGTT) at 16 - 18 weeks' gestation. An OGTT is offered to the other women with risk factors at 24 - 28 weeks (Table 1). However, International Association of Diabetes and Pregnancy Study Groups (IADPSG) recommends universal screening of all pregnant ladies at $24-28$ weeks of gestation [8] [9].

There is not enough evidence to support universal screening as it is not cost effective and could generate more maternal anxiety. Moreover, universal screening will lead to the blanket labelling of a large group of women as having GDM, without differentiating between those at high and low risk of pregnancy related complications [10]. Therefore, in most countries screening of GDM is restricted to only high risk groups to utilize resources appropriately. This practice, however, does carry chance of missing few patients in low risk group. 
Table 1. Diagnostic criteria for gestational diabetes (with 75 g OGTT) [8] [9].

\begin{tabular}{cc}
\hline National institute of clinical excellence (NICE) diagnostic criteria \\
\hline fasting plasma glucose level & $5.6 \mathrm{mmol} /$ litre or above \\
2-hour plasma glucose level & $7.8 \mathrm{mmol} /$ litre or above \\
\hline International Association of Diabetes and Pregnancy Study Groups diagnostic criteria \\
\hline Fasting plasma glucose level & $5.1 \mathrm{mmol} /$ litre or above \\
1 h plasma glucose & $10 \mathrm{mmol} /$ ltre or above \\
2-h plasma glucose level & $8.5 \mathrm{mmol} /$ litre or above \\
\hline
\end{tabular}

\section{Management of GDM}

Appropriate glycaemic control has been a cornerstone in the prevention of maternal and neonatal complications associated with gestational diabetes [11]. Non pharmacological interventions, including diet, exercise and healthy life style are recommended by NICE [7]. It is estimated that $70 \%-90 \%$ of women diagnosed with GDM could achieve targeted glycaemic goals with lifestyle modification and nutrition therapy alone [12]. Dietary advice is based on individual preferences, patient willingness, weight control and blood glucose monitoring.

1) Dietary modification

Dietary recommendations include reduction in amount of simple sugars and replace it with complex carbohydrates to avoid post prandial elevation in blood glucose levels as well as to increase lean protein and vegetables consumption.

Distribution of daily caloric intake should be based on $30 \%-40 \%$ from carbohydrates, $20 \%$ from protein and $40 \%$ from fat [13].

Referral of all women with gestational diabetes to dietitian is recommended by NICE.

2) Exercise

NICE recommends 30 minutes daily walk after meals to achieve good glycaemic control [7]. It is known that exercise increases cells' sensitivity to insulin. Research has shown that the most physically active women have the lowest prevalence of GDM [14].

3) Pharmacological Treatments

Hyperglycaemia and Adverse Pregnancy Outcome (HAPO) trial revealed worse fetal and maternal outcomes if maternal blood glucose is not adequately controlled. Lower glucose levels were associated with better primary outcomes, but there were no obvious thresholds at which the risks increased. Since the HAPO trial, more organizations are recommending lower glucose targets.

If diet and exercise fail to achieve good glycaemic levels, pharmacological therapy is indicated.

4) Insulin therapy

The targets regarding blood glucose levels in gestational diabetes as set by American Diabetes Association (ADA) are: pre-prandial $\leq 95 \mathrm{mg} / \mathrm{dL}(5.3$ $\mathrm{mmol} / \mathrm{L})$ and either 1-hour post-prandial $\leq 140 \mathrm{mg} / \mathrm{dL}(7.8 \mathrm{mmol} / \mathrm{L})$ or 2-hour 
post-prandial $\leq 120 \mathrm{mg} / \mathrm{dL}(6.7 \mathrm{mmol} / \mathrm{L})$ [3]. NICE recommends to consider hypoglycaemic therapy if blood glucose targets are not maintained 1 to 2 weeks after introducing changes to diet and initiating exercise. Insulin therapy can be commenced at very initial presentation in women with severe hyperglycaemia, especially in those patients with postprandial values $>11.1 \mathrm{mmol} / \mathrm{L}$ (>200 $\mathrm{mg} / \mathrm{dL}$ ). Insulin does not cross the placental barrier and therefore is not related with risk of fetal hypoglycaemia.

Long-acting insulin analogues (insulin glargine, insulin detemir) are not recommended, as they have not been studied extensively in pregnancy. For fasting glycaemia, NPH once daily as basal insulin is used at bed time. Post prandial levels can be managed by short acting analogues like insulin aspart. This involves injection at meal time if 1 hour postprandial glucose levels are persistently $>120$ $\mathrm{g} / \mathrm{dl}$ (6.7 mmol/l). The multiple daily dose insulin (Basal-bolus) regimen offers flexibility, precise dose adjustment and consequently better chances of achieving optimal glycaemic control [15]. Insulin is titrated according to the goal of normalising blood sugar. Target glucose levels are below the criteria for insulin initiation (ACOG and ADA criteria) [3].

Although traditionally insulintherapy has been the first line treatment, it is not without challenges. It requires patient motivation, education, self monitoring of glucose, risk of hypoglycaemia, weight gain and needle phobia. Nowadays, there is an option to consider oral hypoglycaemic agents like metformin and glibenclamide before considering insulin therapy.

5) Oral hypoglycaemic agents (OHA)

Many research studies attempted to establish the safety and efficacy of oral hypoglycaemic agents (OHA) [16] [17].

OHAs are less expensive and easier to be administered as compared to insulin therapy. They also seem to be more acceptable by the patients and therefore compliance could be improved. Glyburide and metformin are the drugs which have been studied more extensively [17].

6) Metformin

Metformin lowers serum glucose by inhibiting hepatic gluconeogenesis and glucose absorption and it also stimulates glucose uptake in the peripheral tissues [18]. It has an additional effect in reducing weight gain. It does not cause b cell stimulation and insulin production so hypoglycaemia is not a side effect. It helps to reduce peripheral insulin resistance as well [18]. Unlike insulin, metformin does cross the placental barrier, which previously caused concern for its use in pregnant women. However, studies have shown that transfer of metformin across the placenta does not have negative effects on the fetus [19] [20]. Although it is not licensed for use in GDM, NICE recommends its use if diet and exercise fail to improve glucose control. It can be used on its own or in combination with insulin [7].

The "Metformin in Gestational Diabetes" trial studied 751 women with GDM at 20 - 33 weeks of gestational age, who were randomly assigned to an open treatment of metformin (with supplemental insulin if required). The study was 
designed to compare a range of perinatal complications in women treated with metformin compared to those treated with insulin. Results showed that in women with GDM, metformin (alone or with supplemental insulin) is not associated with increased perinatal complications when compared with the group treated with insulin. The women also preferred metformin to insulin treatment. The study concluded that metformin can be used as an adjunct or an alternate to insulin particularly when there is insulin resistance and requirement of large dose of insulin [16].

A retrospective cohort review of 592 cases which looked for pregnancy outcomes in women with GDM treated with [metformin, lifestyle advice \pm subcutaneous insulin injection] versus [lifestyle advice \pm subcutaneous insulin injection]. The review found no significant difference in maternal and neonatal outcomes between the two groups; however, those in the metformin group had a significantly lower incidence of macrosomia and large for gestational age (LGA) babies.

In general, when compared with insulin, studies show metformin use in pregnancy for treatment of GDM is associated with better glycaemic control, less weight gain and less neonatal hospital admissions. No significant difference in maternal and neonatal outcomes noted except for slightly lower rate of fetalmacrosomia in metformin group [21] [22].

7) Sulfonylurea/Glibenclamide

Sulfonylureas are widely used to treat non-insulin dependent diabetes mellitus. In non pregnant population, these drugs exert their hypoglycaemic effects by stimulating the pancreatic beta-cells. Like metformin, Sulphonylureas are known to cross the placental barrier depending on their molecular mass. Three hours after absorption, tolbutamide attains a cumulative transplacental transfer of $21.5 \%$, chlorpropamide $11 \%$ and glibenclamide (glyburide) only $3.9 \%$ [23]. Glibenclamide is the most commonly used drug of this category in pregnancy and considered as safest option. The safety of other OHG of this class has not been established.

In a randomized controlled trial, a comparison was made regarding the use of glibenclamide and insulin in women with GDM who were unable to achieve adequate metabolic control with diet and exercise alone. The results showed that $82 \%$ of glibenclamide group and $88 \%$ of the insulin group achieved good glycaemic control, but there was less maternal hypoglycaemia in the glibenclamide group (2\%) as compared to the insulin group $(20 \%)$. There were no significant differences between the 2 groups regarding the incidence of fetal or maternal complications [24].

Another study compared glycaemic effect of metformin and glibinclamide as well as the neonatal outcomes. This study showed no significant difference in the mean fasting and 2-hour postprandial blood glucose levels among those who achieved glycaemic control through metformin and glibenclamide therapy. The same study revealed that $34.7 \%$ of women in the metformin group and $16.2 \%$ of those in the glibenclamide group required insulin supplementation. It was also 
reported that the rate of failure to achieve glycaemic control was 2.1 times higher in the metformin group than the glibenclamide group. There was no significant difference in neonatal outcomes in the two groups [25].

NICE currently recommends treatment with glibenclamide for women with gestational diabetes in whom blood glucose targets are not achieved with metformin but who decline insulin therapy or who cannot tolerate metformin [7].

8) Other Oral hypoglycemics

a) Acarbose

It belongs to alpha glucosidase inhibitor group of oral hypoglycemics. It acts by delaying absorption of carbohydrates in gut. As it is not absorbed, it has no systemic effects and it is considered safe during pregnancy. A study by Bertini et al. compared neonatal outcome in gestational diabetes patients treated with insulin, glibenclamide and acarbose. According to the results, there was no statistical difference in the fasting or postprandial plasma glucose levels or in the average newborn weight in the three different groups [26].

b) Roseglitazone

Rosiglitazone is associated with adverse fetal outcome in 2nd and 3rd trimester. Its use is not recommended by NICE. It is classified as category $\mathrm{C}$ by the Food and Drug Authority (FDA) for use in pregnancy [23].

\section{Conclusions}

Early diagnosis and treatment of GDM is associated with improved short and long term outcomes for mother and fetus. Although insulin is time tested first line therapy, its use involves practical challenges. Recent evidence is supportive of use of OHA, especially metformin and glibenclamide.

Metformin, when compared with insulin, is associated with less weight gain, better satisfaction and acceptance and with a lower risk of maternal hypoglycaemia.

OHAs could be considered as a safe alternative for treatment of GDM with comparable short term outcomes. They could be particularly useful as first line agents to treat GDM in developing countries where use of insulin may not be possible due to its cost implications and lack of proper patient education. Their use in clinical practice as first line agent is supported by NICE and American Diabetic Association. Finally, more prospective studies including large cohorts of women are needed to elucidate the effectiveness as well as the safety of oral hypoglycaemic agents in the management of GDM.

\section{References}

[1] National Collaborating Centre for Women's and Children's Health (UK) (2008) Diabetes in Pregnancy: Management of Diabetes and its Complications from Preconception to the Postnatal Period. RCOG Press, London.

[2] Martin, K.E., Grivell, R.M., Yelland, L.N. and Dodd, J.M. (2015) The Influence of Maternal BMI and Gestational Diabetes on Pregnancy Outcome. Diabetes Research and Clinical Practice, 108, 508-513. https://doi.org/10.1016/j.diabres.2014.12.015 
[3] American Diabetes Association (2014) Diagnosis and Classification of Diabetes Mellitus. Diabetes Care, 37, S81-S90. https://doi.org/10.2337/dc14-S081

[4] Catalano, P.M. and Ehrenberg, H.M. (2006) The Short- and Long-Term Implications of Maternal Obesity on the Mother and Her Offspring. BJOG, 113, 1126-1133. https://doi.org/10.1111/j.1471-0528.2006.00989.x

[5] Newson, L. (2012) The Basics: Gestational Diabetes. GP 15 Feb. https://www.gponline.com/gestational-diabetes/diabetes/gestational-diabetes/article /1115759

[6] Crowther, C.A., Hiller, J.E., Moss, J.R., McPhee, A.J., Jeffries, W.S., et al. (2005) Effect of Treatment of Gestational Diabetes Mellitus on Pregnancy Outcomes. The New England Journal of Medicine, 352, 2477-2486. https://doi.org/10.1056/NEJMoa042973

[7] NICE Guideline (2015) Diabetes in Pregnancy: Management from Preconception to Postnatal Period.

https://www.nice.org.uk/guidance/ng3/resources/diabetes-in-pregnancy-manageme nt-from-preconception-to-the-postnatal-period-51038446021

[8] International Association of Diabetes and Pregnancy Study Groups Consensus Panel (2010) International Association of Diabetes and Pregnancy Study Groups Recommendations on the Diagnosis and Classification of Hyperglycemia in Pregnancy. Diabetes Care, 33, 676-682. https://doi.org/10.2337/dc09-1848

[9] Expert Committee on the Diagnosis and Classification of Diabetes Mellitus (2003) Report of the Expert Committee on the Diagnosis and Classification of Diabetes Mellitus. Diabetes Care, 26, S5-20.

[10] Berger, H. and Sermer, M. (2009) Counterpoint: Selective Screening for Gestational Diabetes Mellitus. Diabetes Care, 32, 1352-1354. https://doi.org/10.2337/dc09-0361

[11] Muhas, C. and Naseef, P.P. (2017) A Review Article-Gestational Diabetes Mellitus. International Journal of Current Pharmaceutical Research, 9, 1-5.

[12] Lee-Parritz, A. (2011) Contemporary Management of Gestational Diabetes. Current Opinion in Endocrinology, Diabetes and Obesity, 18, 395-400.

https://doi.org/10.1097/MED.0b013e32834cd3c4

[13] American College of Obstetricians and Gynecologists (2013) Practice Bulletin No 137: Gestational Diabetes Mellitus. Obstetrics \& Gynecology, 122, 406-416. https://doi.org/10.1097/01.AOG.0000433006.09219.f1

[14] Dyck, R., Klomp, H., Tan, L.K., Turnell, R.W. and Boctor, M.A. (2002) A Comparison of Rates, Risk Factors and Outcome of Gestational Diabetes between Aboriginal and Non Aboriginal Women in Saskatoon Health District. Diabetes Care, 25, 487-493. https://doi.org/10.2337/diacare.25.3.487

[15] Blumer, I., Hadar, E., Hadden, D.R., Jovanovic, L., Mestman, J.H., et al. (2013) Diabetes and Pregnancy: An Endocrine Society Clinical Practice Guideline. The Journal of Clinical Endocrinology \& Metabolism, 98, 4227-4249. https://doi.org/10.1210/jc.2013-2465

[16] Rowan, J.A., Hague, W.M., Gao, W., Battin, M.R., Moore, M.P., et al. (2008) Metformin versus Insulin for the Treatment of Gestational Diabetes. The New England Journal of Medicine, 358, 2003-2015. https://doi.org/10.1056/NEJMoa0707193

[17] Nicholson, W., Bolen, S., Witkop, C.T., Neale, D., Wilson, L., et al. (2009) Benefits and Risks of Oral Diabetes Agents Compared with Insulin in Women with Gestational Diabetes: A Systematic Review. Obstetrics \& Gynecology, 113, 193-205. https://doi.org/10.1097/AOG.0b013e318190a459 
[18] Hundal, R.S., Krssak, M., Dufour, S., Laurent, D., Lebon, V., et al. (2000) Mechanism by Which Metformin Reduces Glucose Production in Type 2 Diabetes. Diabetes, 49, 2063-2069. https://doi.org/10.2337/diabetes.49.12.2063

[19] Dhulkotia, J.S., Ola, B., Fraser, R. and Farrell, T. (2010) Oral Hypoglycemic Agents vs. Insulin in Management of Gestational Diabetes: A Systematic Review and Metaanalysis. American Journal of Obstetrics \& Gynecology, 203, 457. https://doi.org/10.1016/j.ajog.2010.06.044

[20] Menato, G., Bo, S., Signorile, A., Gallo, M.L., Cotrino, I., et al. (2008) Current Management of Gestational Diabetes Mellitus. Expert Review of Obstetrics \& Gynecology, 3, 73-91. https://doi.org/10.1586/17474108.3.1.73

[21] Balani, J., Hyer, S.L., Rodin, D.A. and Shehata, H. (2009) Pregnancy Outcomes in Women with Gestational Diabetes Treated with Metformin or Insulin: A Case-Control Study. Diabetic Medicine, 26, 798-802. https://doi.org/10.1111/j.1464-5491.2009.02780.x

[22] Rai, L., Meenakshi, D. and Kamath, A. (2009) Metformin-A Convenient Alternative to Insulin for Indian Women with Diabetes in Pregnancy. Indian Journal of Medical Sciences, 63, 491-497. https://doi.org/10.4103/0019-5359.58878

[23] Elliott, B.D., Schenker, S., Langer, O., Johnson, R. and Prihoda, T. (1994) Comparative Placental Transport of Oral Hypoglycemic Agents in Humans: A Model of Human Placental Drug Transfer. American Journal of Obstetrics \& Gynecology, 171, 653-660. https://doi.org/10.1016/0002-9378(94)90078-7

[24] Langer, O., Conway, D.L., Berkus, M.D., Xenakis, E.M. and Gonzales, O. (2000) A Comparison of Glyburide and Insulin in Women with Gestational Diabetes Mellitus. The New England Journal of Medicine, 343, 1134-1138. https://doi.org/10.1056/NEJM200010193431601

[25] Moore, L.E., Clokey, D., Rappaport, V. and Curet, L.B. (2010) Metformin Compared with Glyburide in Gestational Diabetes: A Randomized Controlled Trial. $O b$ stetrics and Gynecology, 115, 55-59. https://doi.org/10.1097/AOG.0b013e3181c52132

[26] Bertini, A.M., Silva, J.C., Taborda, W., Becker, F., LemosBebber, F.R., et al. (2005) Perinatal Outcomes and the Use of Oral Hypoglycaemic Agents. Journal of Perinatal Medicine, 33, 519-523. https://doi.org/10.1515/JPM.2005.092 